\title{
N-terminally His-tagged hepatitis B core antigens : construction, expression, purification and antigenicity
}

\begin{abstract}
The core antigen of the hepatitis $\mathrm{B}$ virus $(\mathrm{HBcAg})$ has been used widely as a diagnostic reagent for the identification of the viral infection. However, purification using the conventional sucrose density gradient ultracentrifugation is time consuming and costly. To overcome this, HBcAg particles displaying His-tag on their surface were constructed and produced in Escherichia coli. The recombinant His-tagged HBcAgs were purified using immobilized metal affinity chromatography. Transmission electron microscopy and enzymelinked immunosorbent assay (ELISA) revealed that the displayed His-tag did not impair the formation of the core particles and the antigenicity of $\mathrm{HBcAg}$.
\end{abstract}

Keyword: Hepatitis B capsid; Protein display; His-tag; Purification; Antigenicity 